\title{
Preoperative cardiac risk assessment in geriatric patients with hip fractures: an orthopedic surgeons' perspective
}

\author{
C. W. Siu • N. C. H. Sun • T. W. Lau • K. H. Yiu • \\ F. Leung $\cdot$ H. F. Tse
}

Received: 18 August 2010 / Accepted: 31 August 2010

(C) The Author(s) 2010. This article is published with open access at Springerlink.com

\begin{abstract}
Hip fracture is one of the most common orthopedic conditions and is associated with significant morbidity and mortality. With a progressively aging population, the annual incidence of hip fracture is expected to increase substantially. Emerging evidence suggests that early surgery $(<24 \mathrm{~h})$ minimizes complications secondary to immobilization, including orthostatic pneumonia and venous thromboembolism. Delayed surgical repair ( $>48 \mathrm{~h}$ ) has been consistently demonstrated to be associated with an increased risk of 30-day and 1-year mortality. Nonetheless, early surgery necessitates a shorter time for preoperative medical preparation, in particular cardiac assessment. Patients who undergo emergent orthopedic surgery are therefore at greater risk of perioperative cardiac events than those who undergo elective surgery. In addition, the prompt triage system for preoperative cardiac assessment
\end{abstract}

Electronic supplementary material The online version of this article (doi:10.1007/s00198-010-1393-0) contains supplementary material, which is available to authorized users.

C. W. Siu $(\bowtie) \cdot$ K. H. Yiu $\cdot$ H. F. Tse

Cardiology Division, Department of Medicine,

Queen Mary Hospital, The University of Hong Kong,

Hong Kong, China

e-mail: cwdsiu@hkucc.hku.hk

C. W. Siu $\cdot$ H. F. Tse

Research Center of Heart, Brain, Hormone, and Healthy Aging,

The University of Hong Kong,

Hong Kong, China

N. C. H. Sun

Department of Anesthesiology, Queen Mary Hospital,

The University of Hong Kong,

Hong Kong, China

T. W. Lau $\cdot$ F. Leung

Department of Orthopedics and Traumatology,

The University of Hong Kong,

Hong Kong, China not only identifies patients at high risk of perioperative cardiac complications but also reduces unnecessary cardiac consultations for low-risk patients. We review the current recommendations for preoperative cardiac assessment adapted for patients with hip fracture and describe our current triage system for preoperative cardiac consultation.

\section{Keyword Osteoporosis}

\section{Introduction}

Hip fracture is one of the most common orthopedic conditions that requires hospital admission and is associated with significant morbidity and mortality. The annual incidence of hip fracture was estimated to be 1.66 million worldwide in 1990 and is expected to reach 6.26 million by 2050 due to the aging population [1]. The majority of hip fractures occur in geriatric patients: approximately $80 \%$ of women and $50 \%$ of men with hip fractures are aged $\geq 70$ years [2]. More importantly, up to one third of patients will die within 1 year of sustaining a hip fracture repair [3-6], and half will have permanent loss of function [7]. Early surgery $(<24 \mathrm{~h})$ can minimize complications secondary to immobilization including orthostatic pneumonia and venous thromboembolism and is expected to be beneficial for the majority of patients with a fractured hip. Delayed surgery ( $>48 \mathrm{~h}$ ) has been consistently demonstrated by several studies to be associated with an increased risk of 30-day and 1-year mortality [8]. It is thus generally recommended that patients with hip fracture should undergo surgery as soon as possible (within $24 \mathrm{~h}$ of admission), during standard daytime working hours, including weekends, and provided they are medically fit [9].

Minimizing the time between admission and surgery nonetheless allows less time to evaluate and optimize patient's 
underlying medical conditions. While this is not a concern for young individuals with no underlying medical problems, most patients with a hip fracture are frail and elderly with multiple pre-existing medical conditions that warrant comprehensive preoperative evaluation by physicians and/or cardiologists [10]. The goals of preoperative assessment should be (1) to identify patients at high risk of perioperative cardiac events and (2) to reduce their risks of complications and mortality. The American College of Cardiology (ACC) and the American Heart Association (AHA) guidelines for perioperative cardiovascular evaluation for non-cardiac surgery published in 2007 are invaluable protocols for cardiologists; nonetheless, it does not alert primary clinicians as to when a cardiac consultation is required. As a result, orthopedic surgeons, often the key member of the team, may face a clinical dilemma: to injudiciously consult a cardiologist for all elderly patients with a hip fracture, to proceed to timely surgery without a comprehensive preoperative cardiac assessment, or to delay surgery until a cardiac evaluation is complete. Based on the published international guidelines, we present a clinical protocol for preoperative cardiac assessment tailored for the geriatric patient with hip fracture from an orthopedic surgeon's perspective.

\section{Surgical risk of hip fracture repair}

The nature of the surgery, including urgency, magnitude, type, and duration of the operation, is an important determinant in perioperative cardiac complications as well as in mortality. In general, the estimated cardiac risk of major orthopedic surgeries including hip and spine surgery is intermediate, i.e., estimated 30-day cardiac event rate (cardiac death and myocardial infarction) of $1-5 \%$ [11]. This stratification is based on the premise that most orthopedic procedures are electively performed in relatively young, healthy patients. In a stark contrast, elderly patients with a hip fracture who undergo surgical repair often have known predictors of cardiac disease, and the procedure performed is semi-urgent, not elective $(<24 \mathrm{~h})$. The risk profile thus differs. In a retrospective study of 8,930 patients aged $\geq 60$ years who underwent hip fracture repair [12], 30-day and 1-year mortality was $4 \%$ and $16 \%$, respectively. Of the, 720 patients $(8 \%)$ with postoperative cardiac complications, 178 patients $(2 \%)$ were considered to have serious postoperative cardiac complications.

\section{Stepwise approach to preoperative cardiac assessment}

In 2007, the ACC and the AHA published a stepwise approach to preoperative cardiac assessment for patients undergoing non-cardiac surgery [11]. The guidelines consist of five decision-making steps that take into account urgency and risk of the surgery, presence of an active cardiac condition, functional capacity of the patient, and the associated cardiovascular risk factors. This helps determine whether to proceed with the planned surgery [11]. As mentioned, hip fracture repair can be considered a nonemergency (but semi-urgent) surgery with a moderate cardiac risk ( $\sim 5 \%$ perioperative cardiac events and mortality); the original five-step approach could then be adapted to a three-step algorithm for this clinical context. Figure 1 depicts the clinical pathway for preoperative cardiac assessment of patients with a hip fracture. In order to determine whether a patient is medically fit for the surgery, patients with a hip fracture should have complete history and physical examination; in addition, chest X-ray and standard 12-lead electrocardiography should be obtained.

Step 1 Does the patient have any active cardiac conditions? (modified from [11])

The ACC/AHA guidelines have identified four groups of active cardiac conditions that signify major perioperative risk for surgery and that warrant preoperative workup (Table 1). Patients with one or more of these active cardiac conditions require further diagnostic evaluation and, possibly, therapeutic intervention. Of note, patients with underlying coronary artery disease are at higher than average risk of perioperative cardiac events. According to the ACCC/AHA guidelines, a coronary artery disease patient is defined as one with a history of myocardial infarction, percutaneous coronary intervention, coronary artery bypass grafting, or coronary arterial luminal obstruction documented by coronary angiography [11]. A patient with stable coronary artery disease and a functional capacity of four metabolic equivalents (METs) or above (Table 2) is considered medically fit for hip fracture repair surgery although elective surgery should be delayed for at least 6 months in patients with recent acute myocardial infarction. In a case series of 11 patients (mean age 78.2 years, female $73 \%$ ) with recent myocardial infarction (3 to 23 days) who underwent hip fracture repair, 1and 6-month mortality was $45.4 \%$ and $63.5 \%$, respectively; the impact of recent ACS on the risk of perioperative cardiovascular events nonetheless remains unknown.

As with coronary artery disease, heart failure is commonly associated with hip fracture. It has recently been shown in a cohort of 5,613 persons from the Cardiovascular Health Study with average follow-up of 11.5 years that patients with heart failure have a much higher incidence of hip 
Fig. 1 Cardiac evaluation and care algorithm for semi-urgent hip repair (adapted from [13]for geriatric hip fracture repair)

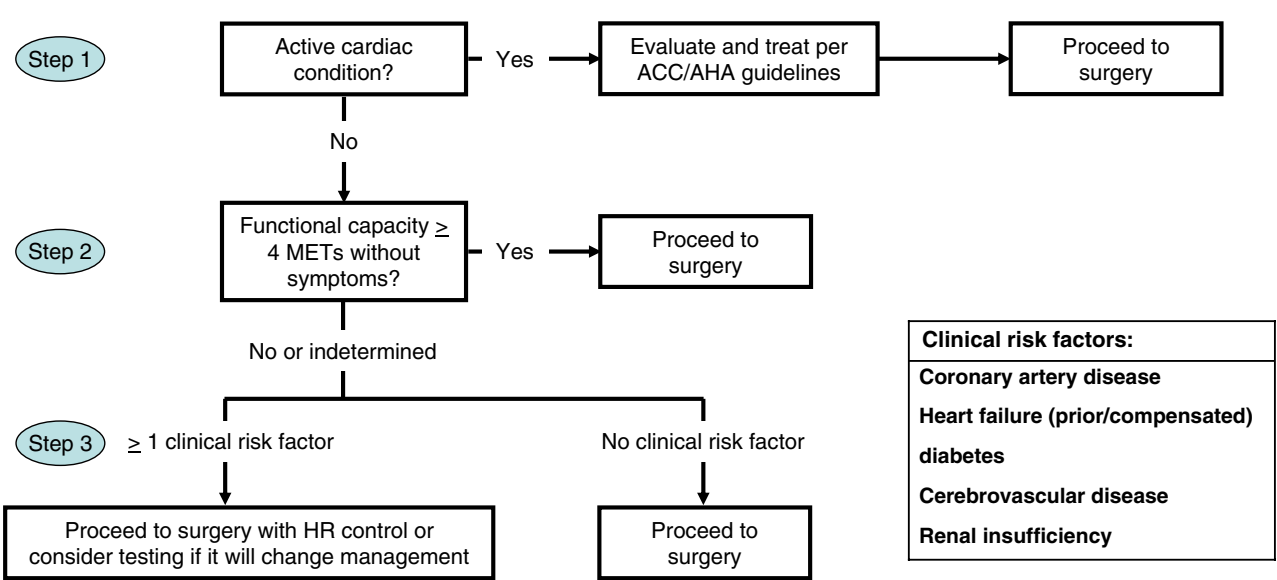

fracture compared with those without heart failure (14/1,000 vs. 6.8/1,000 person-years). More importantly, patients with both heart failure and hip fracture have a twofold increase in risk of death compared with those with heart failure alone [13]. Patients with heart failure who undergo noncardiac surgery have a poorer outcome than those without heart failure [14]. It is thus essential to identify patients with heart failure and optimize their cardiac condition prior to surgery. In addition, the presence of significant valvular disease, in particular, severe aortic stenosis, confers a substantial risk of perioperative cardiac events in

Table 1 Active cardiac conditions (modified from [13])
Unstable coronary syndromes

Significant arrhythmia

Severe valvular disease
Heart failure
Unstable angina

Acute coronary syndrome and/or myocardial infarct

Recent myocardial infarction $(<1$ month)

Decompensated heart failure

NYHA functional class IV

Worsening or new-onset heart failure

Symptomatic bradycardia

Symptomatic sinus bradycardia and/or sick sinus syndrome

High degree atrioventricular block (Mobitz II and third degree heart block)

Supraventricular tachycardia with uncontrolled ventricular rate $(>100 \mathrm{bpm}$ at rest)

Symptomatic ventricular Newly recognized ventricular tachycardia arrhythmia

Severe aortic stenosis Symptomatic mitral stenosis patients who undergo non-cardiac surgery [11, 15-17]. Aortic stenosis is relatively common in geriatric patients $(>65$ years) $[18,19]$ and is often associated with hip fracture. In a retrospective study that included 3,997 consecutive patients with a hip fracture, $272(6.8 \%)$ were confirmed to have a previously undiagnosed aortic stenosis as a result of echocardiography to investigate a previously undiagnosed heart murmur [20]. While it is recommended that echocardiography should be performed as part of a preoperative assessment if aortic stenosis is suspected, to allow confirmation
Table 2 Evaluation of the degree of activity in daily life (modified from [13])

4 METs (degree of activity: low)

If all questions are answered "yes," the patient score is "4 METs"

Otherwise, the score is " $<4$ METs"

Can you take care of yourself? (Eat, dress, use the toilet)?

Can you do light work around the house?

Can you dust or wash dishes by yourself?

Can you walk around and shop in your neighborhood?

Can you do exercise such as gardening?

4-7 METs (degree of activity: moderate)

If all questions are answered "yes," the patient's score is "7 METs"

Otherwise, the score is " $<7$ METs"

Can you walk up a flight of stairs?

Can you walk quickly for a short time (for $3 \mathrm{~min}$, about $300 \mathrm{~m}$ )?

Can you prepare Japanese bedding?

Can you go shopping with heavy bags?

$>7$ METs (degree of activity: good) With more than one "yes" the daily activity score is $>7$ METs.

Can you run or ride a bike uphill?

Can you run for 6 or $7 \mathrm{~min}$ (about $800 \mathrm{~m}$ )?

Can you go up stairs for a distance of two floors?

$>10$ METs (degree of activity: excellent)

If the patient can participate in activities such as swimming, soccer, or skiing, the daily activity score is "> 10 METs" 
of diagnosis, risk stratification, and possible cardiac intervention [21], the clinical decision on whether to operate on such patients remains a challenge due to the scarcity of clinical outcome data. In a retrospective study by Adunsky and colleagues involving 56 patients with hip fracture and aortic stenosis (mean valve area $0.97 \pm$ $0.64 \mathrm{~cm}^{2}$ ) who underwent surgical repair [22], in-hospital and 1-year mortality was $6.5 \%$ and $17.7 \%$, respectively.

Step 2 Does a patient have a functional capacity greater than or equal to 4 METSs without symptoms? (modified from [11])

Table 2 summarizes the estimated energy requirement for various common daily activities. It has been extensively confirmed that a patient's functional status reliably predicts perioperative and long-term cardiac events [23-26]. For asymptomatic patients with a functional capacity of 4 METs or above, the need for any active preoperative cardiac intervention to lower the perioperative risk is unlikely [11].

Step 3 If the patient has poor functional capacity, is symptomatic, or has unknown function, then the presence of clinical risk factors including [1] coronary artery disease [2], compensated heart failure [3], previous cerebrovascular accident [4], diabetes mellitus, and [5] renal insufficiency, will determine the need for further evaluation (modified from [11]).

As hip repair surgery is considered intermediaterisk surgery, even in the presence of risk factors, further cardiac investigations are not generally considered necessary.

While fulfilling these three steps mentioned above provides cardiac clearance for surgery, underlying medical conditions may still warrant medical attention and cardiac consultation, for example, patients with medical assistance devices (permanent pacemaker and automatic implantable cardioverter defibrillator), and those prescribed dual antiplatelet agents or oral anticoagulants.

\section{Clinical pathway for hip fracture management}

While the above-described guidelines provide an invaluable tool for the attending cardiologist to determine perioperative risk for a patient with hip fracture, it does not alert the primary clinician, often an orthopedic surgeon, as to when a cardiac consultation should be initiated. Surgery may be delayed because cardiac clearance cannot be promptly obtained. In order to "fast-track" hip fracture patients for a timely surgery (within the first $24 \mathrm{~h}$ ), a clinical pathway for hip fracture management has been implemented at our hospital since 2008. The frontline orthopedic surgeon and/ or intern evaluates the patient's cardiovascular status according to a checklist (Appendix 1) and determines whether a cardiac consultation is required, even prior to the anesthetist's assessment. As a result, cardiac clearance is usually obtained within the same day. When further investigations, such as echocardiography, are required, they can be scheduled for the following morning. Surgery can still be performed within $24 \mathrm{~h}$ of admission.

\section{Summary}

Hip fracture represents one of the major medical problems faced by our aging society. Early surgery may reduce inhospital, short-term, and long-term morbidity and mortality. Careful screening of patients with hip fracture to enable prompt cardiac assessment can improve overall outcome by minimizing unnecessary delays for cardiac clearance.

Conflicts of interest Dr. Leung is the speaker for Synthes and has received research support from Synthes. None of the other authors has a real or perceived conflict of interest or a disclosure of any personal or financial support.

Open Access This article is distributed under the terms of the Creative Commons Attribution Noncommercial License which permits any noncommercial use, distribution, and reproduction in any medium, provided the original author(s) and source are credited.

\section{References}

1. Cooper C, Campion G, Melton LJ 3rd (1992) Hip fractures in the elderly: a world-wide projection. Osteoporos Int 2:285-289

2. Lauritzen JB, Schwarz P, Lund B, McNair P, Transbol I (1993) Changing incidence and residual lifetime risk of common osteoporosis-related fractures. Osteoporos Int 3:127-132

3. Goldacre MJ, Roberts SE, Yeates D (2002) Mortality after admission to hospital with fractured neck of femur: database study. BMJ 325:868-869

4. Miller CW (1978) Survival and ambulation following hip fracture. J Bone Joint Surg Am 60:930-934

5. Roberts SE, Goldacre MJ (2003) Time trends and demography of mortality after fractured neck of femur in an English population, 1968-98: database study. BMJ 327:771-775

6. Wolinsky FD, Fitzgerald JF, Stump TE (1997) The effect of hip fracture on mortality, hospitalization, and functional status: a prospective study. Am J Public Health 87:398-403

7. Woolf AD, Pfleger B (2003) Burden of major musculoskeletal conditions. Bull World Health Organ 81:646-656

8. Shiga T, Wajima Z, Ohe Y (2008) Is operative delay associated with increased mortality of hip fracture patients? Systematic review, meta-analysis, and meta-regression. Can J Anaesth 55:146-154 
9. Network SIG (2002) Prevention and management of hip fracture in older people: a national clinical guideline. pp 1-40

10. Cooney LM Jr (1997) Hip fracture outcomes. Arch Intern Med 157:485-486

11. Fleisher LA, Beckman JA, Brown KA, Calkins H, Chaikof E, Fleischmann KE, Freeman WK, Froehlich JB, Kasper EK, Kersten JR, Riegel B, Robb JF, Smith SC Jr, Jacobs AK, Adams $\mathrm{CD}$, Anderson JL, Antman EM, Buller CE, Creager MA, Ettinger SM, Faxon DP, Fuster V, Halperin JL, Hiratzka LF, Hunt SA, Lytle BW, Nishimura R, Ornato JP, Page RL, Tarkington LG, Yancy CW (2007) ACC/AHA 2007 guidelines on perioperative cardiovascular evaluation and care for noncardiac surgery: a report of the American college of cardiology/American heart association task force on practice guidelines (writing committee to revise the 2002 guidelines on perioperative cardiovascular evaluation for noncardiac surgery): developed in collaboration with the American society of echocardiography, American society of nuclear cardiology, heart rhythm society, society of cardiovascular anesthesiologists, society for cardiovascular angiography and interventions, society for vascular medicine and biology, and society for vascular surgery. Circulation 116:e418-e499

12. Lawrence VA, Hilsenbeck SG, Noveck H, Poses RM, Carson JL (2002) Medical complications and outcomes after hip fracture repair. Arch Intern Med 162:2053-2057

13. Carbone L, Buzkova P, Fink HA, Lee JS, Chen Z, Ahmed A, Parashar S, Robbins JR (2010) Hip fractures and heart failure: findings from the Cardiovascular Health Study. Eur Heart J 31:77-84

14. Lee TH, Marcantonio ER, Mangione CM, Thomas EJ, Polanczyk CA, Cook EF, Sugarbaker DJ, Donaldson MC, Poss R, Ho KK, Ludwig LE, Pedan A, Goldman L (1999) Derivation and prospective validation of a simple index for prediction of cardiac risk of major noncardiac surgery. Circulation 100:1043-1049

15. Detsky AS, Abrams HB, Forbath N, Scott JG, Hilliard JR (1986) Cardiac assessment for patients undergoing noncardiac surgery. A multifactorial clinical risk index. Arch Intern Med 146:2131-2134
16. Goldman L, Caldera DL, Nussbaum SR, Southwick FS, Krogstad D, Murray B, Burke DS, O’Malley TA, Goroll AH, Caplan CH, Nolan J, Carabello B, Slater EE (1977) Multifactorial index of cardiac risk in noncardiac surgical procedures. N Engl J Med 297:845-850

17. Chambers J (2005) Aortic stenosis. Bmj 330:801-802

18. Lindroos M, Kupari M, Heikkila J, Tilvis R (1993) Prevalence of aortic valve abnormalities in the elderly: an echocardiographic study of a random population sample. J Am Coll Cardiol 21:1220-1225

19. Stewart BF, Siscovick D, Lind BK, Gardin JM, Gottdiener JS, Smith VE, Kitzman DW, Otto CM (1997) Clinical factors associated with calcific aortic valve disease. Cardiovascular health study. J Am Coll Cardiol 29:630-634

20. McBrien ME, Heyburn G, Stevenson M, McDonald S, Johnston NJ, Elliott JR, Beringer TR (2009) Previously undiagnosed aortic stenosis revealed by auscultation in the hip fracture populationechocardiographic findings, management and outcome. Anaesthesia 64:863-870

21. Network SIG (2009) Management of hip fracture in older people. pp $1-48$

22. Adunsky A, Kaplan A, Arad M, Mizrahi EH, Gottlieb S (2008) Aortic stenosis in elderly hip fractured patients. Arch Gerontol Geriatr 46:401-408

23. Bartels C, Bechtel JF, Hossmann V, Horsch S (1997) Cardiac risk stratification for high-risk vascular surgery. Circulation 95:2473-2475

24. Myers J, Do D, Herbert W, Ribisl P, Froelicher VF (1994) A nomogram to predict exercise capacity from a specific activity questionnaire and clinical data. Am J Cardiol 73:591-596

25. Nelson CL, Herndon JE, Mark DB, Pryor DB, Califf RM, Hlatky MA (1991) Relation of clinical and angiographic factors to functional capacity as measured by the Duke activity status index. Am J Cardiol 68:973-975

26. Older P, Hall A, Hader R (1999) Cardiopulmonary exercise testing as a screening test for perioperative management of major surgery in the elderly. Chest 116:355-362 